\title{
An Axin2 mutation and perinatal risk factors contribute to sagittal craniosynostosis: evidence from a Chinese female monochorionic diamniotic twin family
}

Jin Xu ${ }^{1,2,3}$, Qing Yan ${ }^{4}$, Chengcheng Song ${ }^{5}$, Jingjia Liang ${ }^{1,2}$, Liang Zhao ${ }^{6}$, Xin Zhang ${ }^{1,2}$, Zhenkun Weng ${ }^{1,2}$, Cheng $X u^{1,2}$, Qian Liu ${ }^{1,2}$, Shuqin Xu ${ }^{1,2}$, Lu Pang ${ }^{1,2}$, Liye Zhang ${ }^{1,2}$, Yuan Sun ${ }^{2}$, Gang Wang ${ }^{4^{*}}$ and Aihua Gu $u^{1,2^{*}}$

\begin{abstract}
Background: Craniosynostosis, defined as premature fusion of one or more cranial sutures, affects approximately 1 in every 2000-2500 live births. Sagittal craniosynostosis (CS), the most prevalent form of isolated craniosynostosis, is caused by interplay between genetic and perinatal environmental insults. However, the underlying details remain largely unknown.
\end{abstract}

Methods: The proband (a female monochorionic twin diagnosed with CS), her healthy co-twin sister and parents were enrolled. Obstetric history was extracted from medical records. Genetic screening was performed by whole exome sequencing (WES) and confirmed by Sanger sequencing. Functional annotation, conservation and structural analysis were predicted in public database. Phenotype data of Axin2 knockout mice was downloaded from The International Mouse Phenotyping Consortium (IMPC, http://www.mousephenotype.org).

Results: Obstetric medical records showed that, except for the shared perinatal risk factors by the twins, the proband suffered additional persistent breech presentation and intrauterine growth restriction. We identified a heterozygous mutation of Axin2 (c.1181G > A, p.R394H, rs200899695) in monochorionic twins and their father, but not in the mother. This mutation is not reported in Asian population and results in replacement of Arg at residue 394 by His (p.R394H). Arg 394 is located at the GSK3 $\beta$ binding domain of Axin2 protein, which is highly conserved across species. The mutation was predicted to be potentially deleterious by in silico analysis. Incomplete penetrance of Axin2 haploinsufficiency was found in female mice.

Conclusions: Axin2 (c.1181G > A, p.R394H, rs200899695) mutation confers susceptibility and perinatal risk factors trigger the occurrence of sagittal craniosynostosis. Our findings provide a new evidence for the gene-environment interplay in understanding pathogenesis of craniosynostosis in Chinese population.

Keywords: Sagittal craniosynostosis, Axin2, Perinatal risk factors, Mutation, Monochorionic diamniotic twin

\footnotetext{
*Correspondence: aihuagu@njmu.edu.cn; wang509111@163.com

${ }^{1}$ State Key Laboratory of Reproductive Medicine, Institute of Toxicology, Nanjing Medical University, Nanjing 211166, China

${ }^{4}$ Department of Neurosurgery, Children's Hospital of Nanjing Medical University, Nanjing 210017, China

Full list of author information is available at the end of the article
}

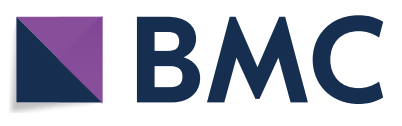

(c) The Author(s) 2021. Open Access This article is licensed under a Creative Commons Attribution 4.0 International License, which permits use, sharing, adaptation, distribution and reproduction in any medium or format, as long as you give appropriate credit to the original author(s) and the source, provide a link to the Creative Commons licence, and indicate if changes were made. The images or other third party material in this article are included in the article's Creative Commons licence, unless indicated otherwise in a credit line to the material. If material is not included in the article's Creative Commons licence and your intended use is not permitted by statutory regulation or exceeds the permitted use, you will need to obtain permission directly from the copyright holder. To view a copy of this licence, visit http://creativecommons.org/licenses/by/4.0/. The Creative Commons Public Domain Dedication waiver (http://creativeco mmons.org/publicdomain/zero/1.0/) applies to the data made available in this article, unless otherwise stated in a credit line to the data. 


\section{Introduction}

Craniosynostosis (CS), defined as premature fusion of one or more cranial sutures, affects approximately 1 in every 2000-2500 live births [1]. CS contains an isolated condition (non-syndromic craniosynostosis, NCS) and complex syndromes (with other malformations, syndromic craniosynostosis) [2]. Sagittal craniosynostosis is the most prevalent form of NCS, accounting for $40-58 \%$ of all NCS cases [3]. Sagittal suture premature closure restricts the widen of the skull and then causes the scaphocephaly deformity and other adverse neurologic outcomes [4].

Pathoetiology of NCS involves interplay between genetic and environmental factors [5-8]. FGF (fibroblast growth factor), BMP (bone morphogenic protein), Wnt (wingless-type integration sites) pathways are major regulators in suture biology $[9,10]$. Pivotal component mutations in these pathways, including FGFR2 (fibroblast growth factor receptor 2), TWIST1 (twist, drosophila, homolog of 1) and Axin2 (axis inhibitor 2), have been regarded as the origin of craniosynostosis [1, 9]. Non-genetic risk factors, like intrauterine constraint, twin gestation, breech delivery, low birth weight, malnutrition, premature delivery, maternal thyroid disorders, gestational diabetes, virus infectious, can either cause or exacerbate craniosynostosis [7, 11-14]. Although several findings demonstrate the interactions between genetic and environmental risk factors contribute to premature fusion of cranial sutures $[5,15]$, more evidence are still need.

Monochorionic (MC) twins, sharing almost the same genome, offer a unique opportunity to study the geneenvironment interactions, for the healthy twin as an ideal control. Discordant phenotypes between MC twins emphasize the interplay between genetic and environmental influences in etiologies of the disease [16].

In our study, we found that a heterozygous mutation of Axin2 (c.1181G>A, p.R394H, rs200899695) exist in Chinese female monochorionic sisters and their father. However, only the proband, suffering a persistent breech presentation and intrauterine growth restriction, was diagnosed with sagittal craniosynostosis. Public database indicated that Axin2 (c.1181G > A, p.R394H, rs200899695) mutation was not detected in Asian population. According to data from The International Mouse Phenotyping Consortium (IMPC, http://www.mousephenotype.org), about 22\% female Axin2 heterozygous knockout mice developed abnormal head shape before delivery. Thus, we speculate that this particular Axin2 mutation leads to haploinsufficiency in female with incomplete penetrance, and additional environmental insults eventually trigger the occurrence of sagittal craniosynostosis.

\section{Materials and methods} Clinic Examination and Information

All participants signed the informed consent and received physical examination by two experienced surgeons in Children's Hospital of Nanjing Medical University. All samples used in our study were in compliance with the informed consent and agreement of patients. This study was approved by the ethics committee of Nanjing Medical University.

Clinical information of pregnancy history and infant clinical data were obtained from hospital medical records. Head CT scan of the healthy child was performed upon the request of the parents.

\section{Whole Exome Sequencing and Data Analysis}

Genomic DNA, extracted from peripheral blood samples (proband, proband's co-twin sister, the parents) and proband's skull periosteum tissue, was subjected to whole-exome sequencing (WES) on the platform of Genergy Biotechnology, Shanghai, China. Raw reads were aligned to the human genome reference assembly (GRCh37/hg19) using the Burrows-Wheeler Aligner [17]. The Picard software was employed to remove PCR duplicates and evaluate the quality of variants. DNA variants was called and analyzed using the Genome Analysis Toolkit [18]. The variants with read depths less than $4 \times$ were filtered out. All variants were further annotated [19-25]. The workflow of genetic analysis was shown in Fig. 1.

\section{Sanger Sequencing}

Axin 2 mutation was validated by Sanger sequencing in Tsingke (China) using the following primers: Forward: 5'-CGCACACCCTAACGCACCCCAT-3' and Reverse: $5^{\prime}$-ACCGCCCACCTAGCCTGCTGAA- ${ }^{\prime}$. Results were visualized using FinchTV (Geospiza) software.

\section{Conservation Analysis}

Multiple-species amino acid sequences were obtained from National Center for Biotechnology Information (NCBI), and were analyzed by WebLogo (Version 2.8.2, http://weblogo.berkeley.edu)[26].

\section{Structural Analysis}

Three-dimensional models of the wild-type and mutant Axin 2 protein were constructed by I-TASSER [27] and visualized using the PyMOL software (PyMOL Molecular Graphics System, DeLano Scientific, San Carlos, CA). 


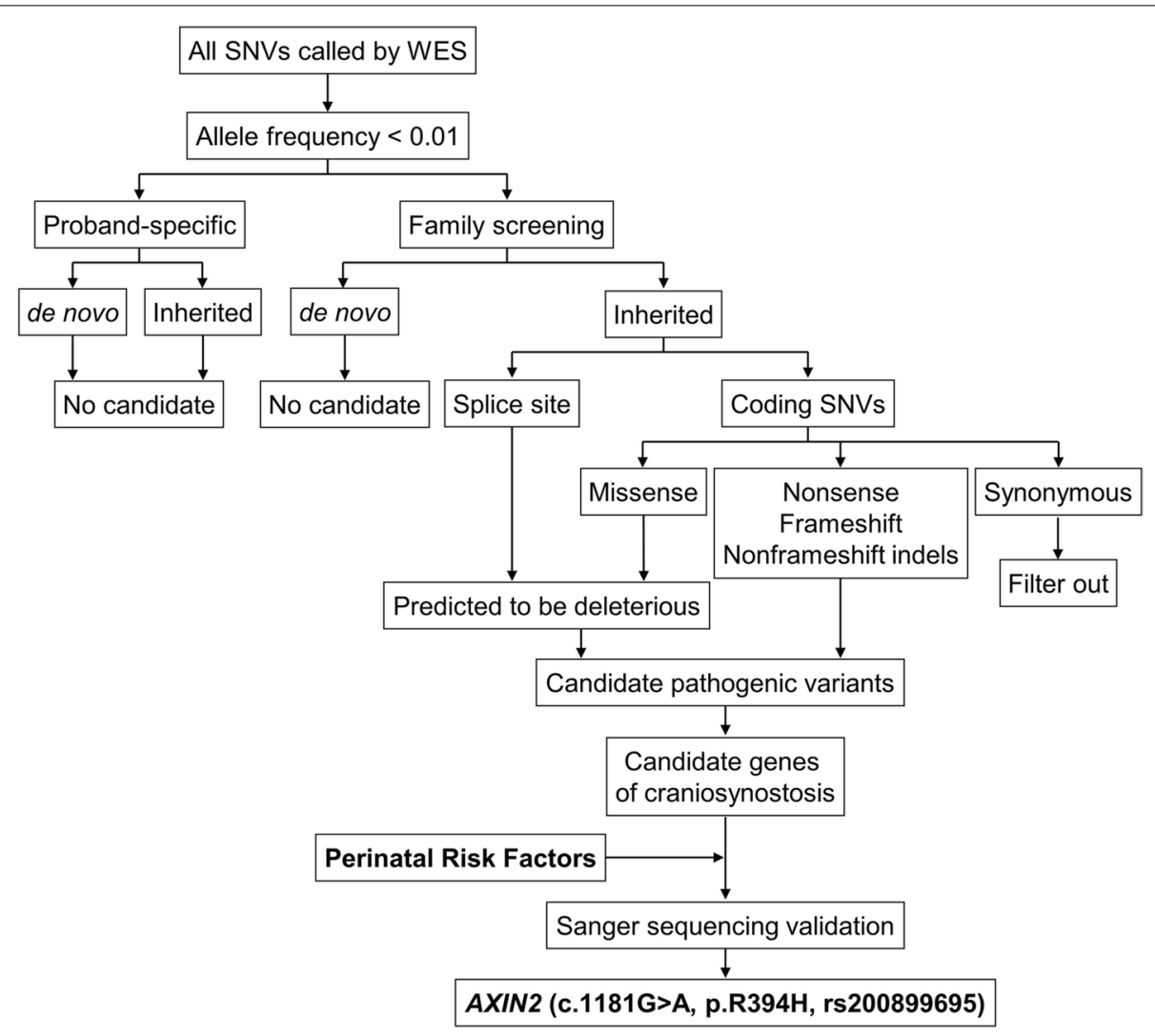

Fig. 1 Workflow for identifying Axin2 (c.1181G > A, p.R394H, rs200899695) mutation in pedigree diagnosed with sagittal craniosynostosis

\section{Functional Annotation}

Functional annotation of Axin2 (c.1181G > A: p.R394H, rs200899695) was conducted on FAVOR functional annotation online portal (http://www.favor.genohub. org/) [28] or the University of California, Santa Cruz Genome Browser (UCSC, http://www.genome.ucsc.edu).

\section{Phenotype analysis of Axin2 knockout mice}

Phenotypes of $A x i n 2$ homozygous $\left(A x i n 2^{-1-}\right)$ and heterozygous $\left(A x i n 2^{+/-}\right)$knockout mice were obtained from The International Mouse Phenotyping Consortium (IMPC, http://www.mousephenotype.org) [29, 30].
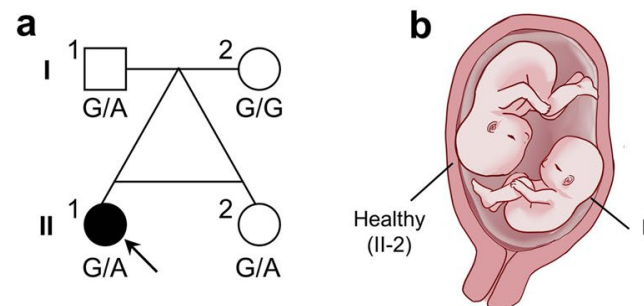

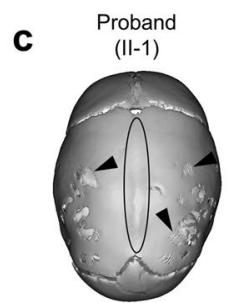

Cranial index $=70.9 \%$ Cranial index $=83.2 \%$

Fig. 2 Clinical features of the pedigree with sagittal craniosynostosis. a Pedigree of the family. Proband is marked with an upward arrow. Open squares/circles denote unaffected individuals; squares denote males and circles denote females. $\mathbf{b}$ Schematic diagram of fetal position before delivery. Proband (II-1) was in breech presentation at left side of the uterus, while her healthy sister (II-2) was in cephalic presentation at right side of the uterus. c Computerized tomographic (CT) scan revealed premature closure of sagittal suture (solid circle) and digital impressions (arrowheads) in proband's head (left panel). Cranial index of the proband is $70.9 \%$. The co-twin sister was also received CT examination under the request of the parents. Her sagittal suture remains patent (dotted circle) and cranial index is $83.2 \%$ (right panel) 

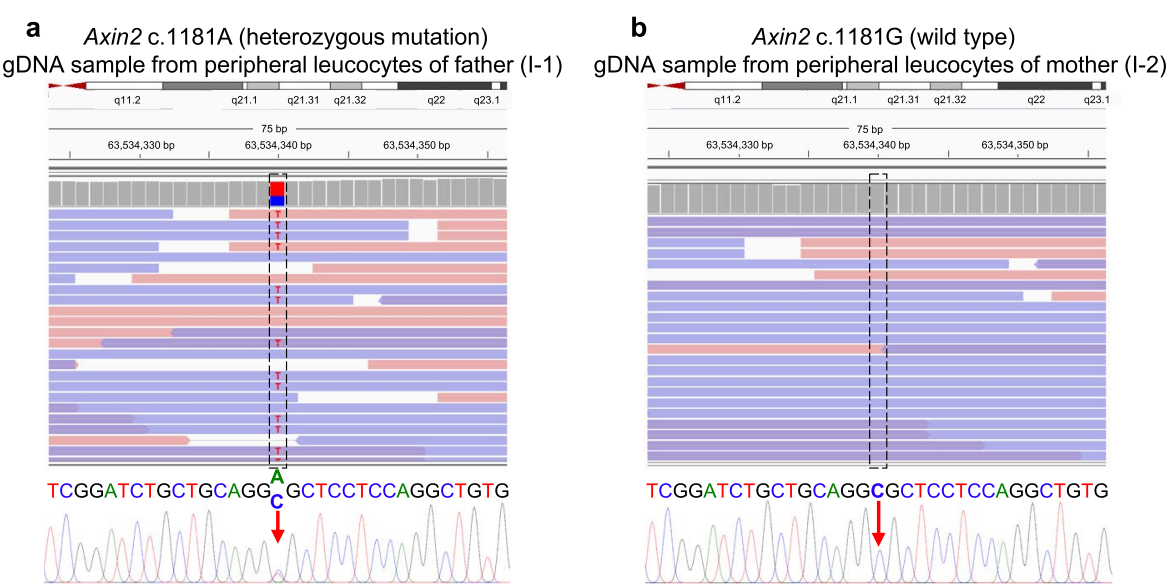

C Axin2 c.1181A (heterozygous mutation) gDNA sample from peripheral leucocytes of sister (II-2)

\section{d}

Axin2 c.1181A (heterozygous mutation)

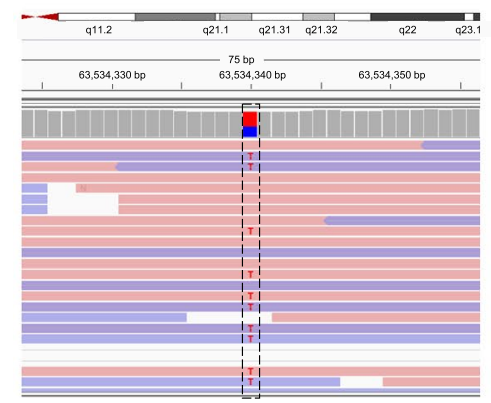

TCGGATCTGCTGCAGG ${ }_{\mathrm{C}}^{\text {ACTCCTCCAGGCTGTG }}$ Whathonltant

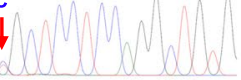

gDNA sample from peripheral leucocytes of proband (II-1)

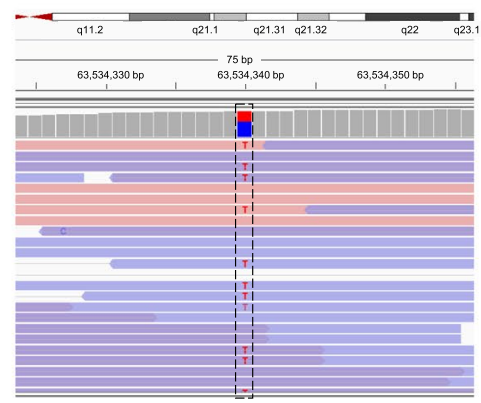

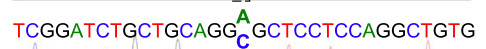

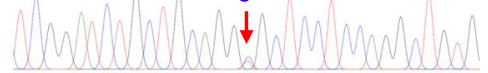

e $\quad$ Axin2 c.1181A (heterozygous mutation) gDNA sample from skull periosteum of proband (II-1)

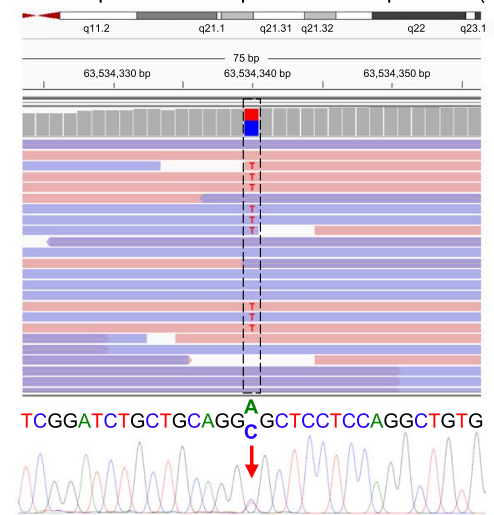

Fig. 3 Sequence analysis of Axin2 (c.1181G >A, p.R394H, rs200899695). a-e Integrative Genomics Viewer (IGV) of the sequences around Axin2 (c.1181G > A, p.R394H, rs200899695) and the results of Sanger sequencing were shown. The gDNA samples were extracted from peripheral leucocytes of the parents and twin sisters (a-d) and from skull periosteum tissue of the proband (e). Please note that the DNA sequences are shown in + strand, but Axin2 is located on-strand. The father and two twins have this missense mutation $(\mathbf{a}, \mathbf{c}, \mathbf{d}, \mathbf{e})$, but the mother does not carry this mutation (b)

\section{Results}

\section{Clinical information}

The pedigree came from Wuhan, Hubei Province,
China. Female proband (II-1), the elder monochorionic diamniotic (MCDA) twin (Fig. 2a-c and Supplementary Fig. S1-3), was diagnosed sagittal craniosynostosis 
at the age of 9 months in the Department of Neurosurgery, Children's Hospital of Nanjing Medical University. She was born to non-consanguineous parents without family history of craniosynostosis. Her mother, a 30-year-old Chinese female, conceived MCDA twins by frozen embryo transfer (FET) (Supplementary Fig. S1-3). Her father was 36-year-old at that time. Twentysix days after FET, two embryos inside a gestational sac (approximately $22 \mathrm{~mm} \times 13 \mathrm{~mm}$ ) was confirmed by four-dimensional ultrasound scan (Supplementary Fig. S1). Embryo length were $2.8 \mathrm{~mm}$ and $3.3 \mathrm{~mm}$, respectively. Fetal heart rate were 107 per minute and 118 per minute, respectively (Supplementary Fig. S1).

At 13 weeks of gestation, IgG of cytomegalovirus and herpes simplex virus were detected in the mother's serum (Supplementary Table S1). At 14 weeks of gestations, dietary assessment indicated that the mother had inadequate intakes of energy, protein, fat, several vitamins and minerals, while excessive consumption of carbohydrate (Supplementary Table S2). At 17 weeks of gestations, decreased thyroid-stimulating hormone (TSH) level and increased level of urinary iodine were detected (Supplementary Table S3). At 27 weeks of gestations, the mother was diagnosed with gestational diabetes mellitus (Supplementary Table S3).

The ultrasound scan detection indicated that the proband (II-1) had been in persistent breech position on the left side of uterus, while the younger sister (II-2) had been in cephalic position (Fig. 2a, b). At 28 weeks of gestations, the proband (II-1) and twin younger sister (II-2) were born via spontaneous vaginal delivery (Fig. 2a, b). Birth weight of proband (II-1) and co-twin (II-2) was $880 \mathrm{~g}$ (ranking $50^{\text {th }}-90^{\text {th }}$ centiles for postnatal weight of infants [31]) and $990 \mathrm{~g}$ (ranking $90^{\text {th }}-97^{\text {th }}$ centiles for postnatal weight of infants [31]), respectively. The proband (II-1) were diagnosed with sagittal craniosynostosis and intrauterine growth restriction (Fig. 2c), while no signs of craniofacial deformity were detected in parents (I-1, I-2) and co-twin sister (II-2) (Fig. 2c). Cranial index, which represents the ratio of maximum cranial width to maximum cranial length, is decreased in patients with sagittal craniosynostosis [32]. In our case, the cranial index of the proband and co-twin sister was $70.9 \%, 83.2 \%$, respectively. In addition, tooth agenesis, oral clefts or colorectal cancer were not detected in this family through physical examination by clinical doctor as well as medical history inquiry. And the other family members have no history of tooth agenesis, oral clefts or colorectal cancer. Taken together, clinical records indicate that the proband suffered sagittal craniosynostosis, persistent breech presentation and intrauterine growth restriction, except for other shared perinatal risk factors of the twins.

Table 1 Allele frequency of Axin2 (c.1181G >A: p.R394H, rs200899695) in human populations (dbGaP, Release Version: $20,200,227,123,210)$

\begin{tabular}{|c|c|c|c|c|c|c|}
\hline Study & Population & Sample size & Ref Allele & Alt Allele & BioProject ID & BioSample ID \\
\hline gnomAD-Exomes & Global & 250,890 & $G=0.999151$ & $A=0.000849$ & PRJNA398795 & SAMN07488253 \\
\hline gnomAD_Exomes & European & 134,850 & $G=0.999993$ & $A=0.000007$ & & SAMN10181265 \\
\hline gnomAD_Exomes & Asian & 49,008 & $\mathrm{G}=1.00000$ & $A=0.00000$ & & \\
\hline gnomAD_Exomes & American & 34,584 & $G=0.99396$ & $A=0.00604$ & & SAMN07488255 \\
\hline gnomAD_Exomes & African & 16,244 & $G=1.00000$ & $A=0.00000$ & & SAMN07488254 \\
\hline gnomAD_Exomes & Ashkenazi Jewish & 10,072 & $G=1.00000$ & $A=0.00000$ & & SAMN07488252 \\
\hline gnomAD_Exomes & Other & 6132 & $G=0.9995$ & $A=0.0005$ & & SAMN07488248 \\
\hline ExAC & Global & 120,894 & $G=0.999363$ & $A=0.000637$ & PRJEB8661 & SAMN07490465 \\
\hline EXAC & Europe & 72,950 & $G=0.99999$ & $A=0.00001$ & & \\
\hline EXAC & Asian & 25,152 & $G=1.00000$ & $A=0.00000$ & & \\
\hline EXAC & American & 11,542 & $G=0.99342$ & $A=0.00658$ & & \\
\hline EXAC & African & 10,358 & $G=1.00000$ & $A=0.00000$ & & \\
\hline EXAC & Other & 892 & $G=1.000$ & $A=0.000$ & & SAMN07486028 \\
\hline ALFA & Total & 62,874 & $G=0.99992$ & $A=0.00008$ & PRJNA507278 & SAMN10492705 \\
\hline ALFA & European & 59,864 & $G=0.99995$ & $A=0.00005$ & & SAMN10492695 \\
\hline ALFA & Other & 2654 & $G=0.9992$ & $A=0.0008$ & & SAMN1 1605645 \\
\hline ALFA & African & 242 & $G=1.000$ & $A=0.000$ & & SAMN10492703 \\
\hline ALFA & Asian & 78 & $G=1.00$ & $A=0.00$ & & SAMN10492704 \\
\hline ALFA & Latin American 2 & 22 & $G=1.00$ & $A=0.00$ & & SAMN10492700 \\
\hline ALFA & South Asian & 8 & $\mathrm{G}=1.0$ & $A=0.0$ & & SAMN10492702 \\
\hline ALFA & Latin American 1 & 6 & $\mathrm{G}=1.0$ & $A=0.0$ & & SAMN10492699 \\
\hline
\end{tabular}




\section{Mutation analysis of AXIN2 (c.1181G > A, p.R394H, rs200899695)}

Whole-exome sequencing was applied to identify the potential genetic etiology leading to sagittal craniosynostosis in our case. Due to the low incidence rate of sagittal craniosynostosis [3], we focused on private and/or rare (minor allele frequency, $\mathrm{MAF}<0.01$ ) variants on exons or splicing sites (Fig. 1). However, none of candidate germline or somatic mutations were proband-specific. As gene-environment interactions have been demonstrated in the pathogenesis of craniosynostosis [5, 15], we wonder whether the intrauterine risk exposures (environment factors) triggered the susceptible individual to develop sagittal craniosynostosis. Based on this hypothesis, we re-analyzed our sequencing data and identified a heterozygous missense mutation of Axin2 (c.1181G > A, p.R394H, rs200899695) in the leukocytes of subjects I-1, II-1 and II-2, and skull periosteum tissue of subject II-1. This finding was further validated by Sanger sequencing (Fig. 3a-e). The frequency of Axin2 (c.1181G >A, p.R394H, rs200899695) mutation of global population is 0.000849, 0.000637, 0.00008 in gnomAD, ExAC, ALFA database, respectively, while is not detected in Asian population (Table 1).

\section{Conservation analysis of Axin2 (c.1181G > A, p.R394H, rs200899695)}

G to A transition of Axin2 (c.1181G >A, p.R394H, rs200899695) resulted in the replacement of Arg by His at $394^{\text {th }}$ AXIN2 protein residue (Fig. 4a). Arg394 residue, located at the GSK3 $\beta$ binding domain (amino acid 327 to 413 according to the UniProt Consortium) of Axin2 protein (Fig. 4a) [33], is conserved across species (Fig. 4b,c and Table 2).

\section{Functional annotation and structural analysis and of Axin2} (c.1181G > A: p.R394H, rs200899695)

Axin2 (c.1181G > A: p.R394H, rs200899695) mutation is predicted to be potentially deleterious by in silico analysis (Table 3) and the wild type Axin2 (c.1181G) loci is located within a region modified by $\mathrm{H} 3 \mathrm{~K} 4 \mathrm{Me} 3$ and H3K27Ac (Fig. 5 a). In addition, p. R394H substitution is predicted to affect spatial structure of AXIN2 GSK3 $\beta$ binding domain (Fig. 5 b, c).

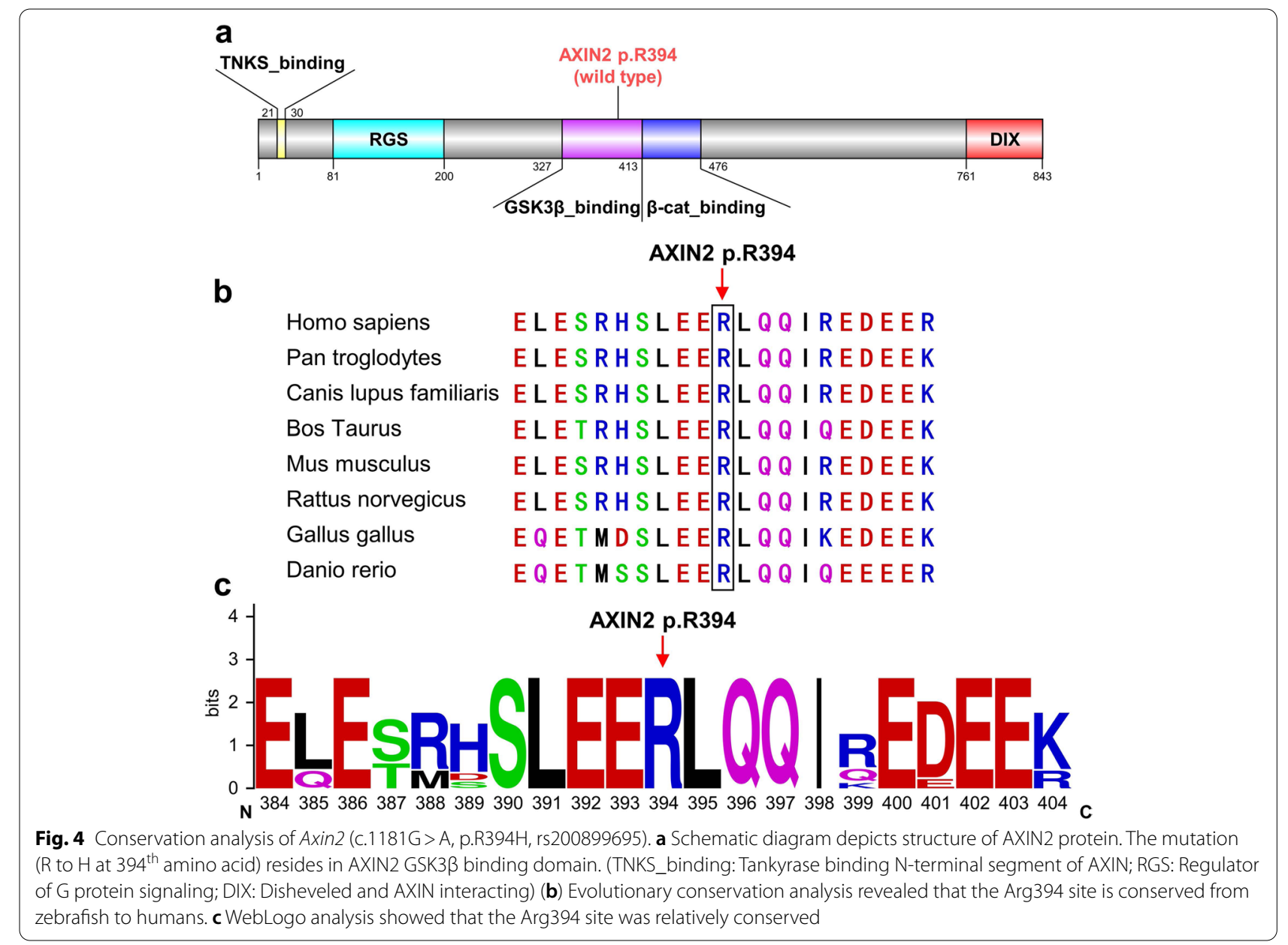


Table 2 Conservation prediction of AXIN2 (c.1181G > A: p.R394H, rs200899695)

\begin{tabular}{lll}
\hline Conservation prediction & Value & Range $^{\text {a }}$ \\
\hline priPhCons & 0.99 & $0-0.999$ (default: 0.0) \\
mamPhCons & 0.97 & $0-1$ (default: 0.0) \\
verPhCons & 1 & $0-1$ (default: 0.0) \\
priPhyloP & 0.42 & $-10.761-0.595$ (default: -0.029$)$ \\
mamPhyloP & 2.75 & $-20-4.494$ (default: -0.005$)$ \\
verPhyloP & 4.82 & $-20-11.295$ (default: 0.042$)$ \\
GerpN & 16.5 & $0-19.8$ (default: 3.0$)$ \\
GerpS & 12.1 & $-39.5-19.8$ (default: -0.2$)$ \\
\hline
\end{tabular}

${ }^{a}$ A higher score means the region is more conserved

\section{Phenotype analysis of Axin2 knockout mice}

Data from The International Mouse Phenotyping Consortium (IMPC) documented that homozygous Axin2 knockout $\left(A x i n 2^{-/-}\right)$mice developed preweaning lethality, while heterozygous $A x i n 2$ knockout $\left(A x i n 2^{+/-}\right)$was alive (Fig. 6 a). The percentage of abnormal craniofacial morphology at embryonic day 12.5 (E12.5) for female Axin $2^{+/+}$and Axin $2^{-/-}$mice was $0.73 \%$ (4/547) and 50\% $(1 / 2)$, respectively (Fig. 6 b). In addition, all female $(6 / 6)$

Table 3 Functional annotation of AXIN2 (c.1181G > A: p.R394H, rs200899695)

\begin{tabular}{ll}
\hline Block/Annotation Name & Data \\
\hline ClinVar & germline \\
Allele Origin & \\
Variant Category & Yes \\
Disruptive Missense ${ }^{a}$ & Yes \\
GeneHancer ${ }^{b}$ & Yes \\
SuperEnhancer ${ }^{c}$ & \\
Protein Function & Probably damaging \\
Polyphen2_HDIV & possibly damaging \\
Polyphen2_HVAR & Disease causing \\
MutationTaster & Deleterious \\
LRT & Deleterious \\
SIFT & predicted functional (medium) \\
MutationAssessor & Deleterious \\
FATHMM & Deleterious \\
PROVEAN & Deleterious \\
MetaSVM & Deleterious \\
MetaLR & Deleterious \\
M-CAP & Deleterious \\
CADD_phred & Deleterious \\
Fathmm-MKL_coding &
\end{tabular}

a Defined as "disruptive" by the ensemble MetaSVM annotation

b Predicted human enhancer sites from the GeneHancer database

c Predicted super-enhancer sites and targets in a range of human cell types and male (3/3) Axin2 $2^{-/-}$mice had abnormal head shape, whereas $22.22 \%(2 / 9)$ female $A x i n 2^{+/-}$mice were with abnormal head shape at E18.5 (Fig. 6 c). However, none of male (0/5) Axin2 $2^{+/-}$mice presented with abnormal head shape (Fig. 6 c). Taken together, these results indicate incomplete penetrance of Axin2 haploinsufficiency in female mice.

\section{Discussion}

Craniosynostosis, a highly heterogeneous disease, is caused by genetic mutations, adverse environmental exposures and their interactions. Identifying the pathoetiology of craniosynostosis gives light to uncover susceptibility individuals, discern environmental risk factors and establish effective strategies for prevention and early diagnosis. In our study, we demonstrated that a heterozygous Axin2 (c.1181G > A: p.R394H, rs200899695) mutation was presented in the monochorionic twins and their father, but not in the mother. However, only the female proband, who was received additional environmental insults (persistent breech presentation and intrauterine growth restriction), developed sagittal craniosynostosis. We assume that this Axin2 mutation predisposes to sagittal craniosynostosis but extra environmental insults are needed to initiate the disease.

Prenatal risk factors, including intrauterine constraint, twin gestation, premature delivery, maternal thyroid disorders, gestational diabetes, malnutrition, virus infectious, increase the susceptibility of craniosynostosis in genetically predisposed infants [7, 11-14]. Research of monochorionic (MC) twins provide exceptional opportunity to decipher the interplay between genetic and environment risks on the occurrence of premature suture fusion [34]. In our study, monochorionic twins suffered the majority of risk factors prenatally, however, only the infant with breech presentation and intrauterine growth restriction presented sagittal craniosynostosis. Thus, intrauterine growth restriction and breech position deserves particularly attention in causing sagittal craniosynostosis.

It has been well accepted that AXIN2 is essential for normal calvarial morphogenesis by directly targeting $\beta$-catenin, orchestrating the crosstalk of Wnt, BMP, FGF signaling pathways and maintaining suture cell stemness [35-37]. Deletion or mutation of Axin2 attribute to craniosynostosis in humans and mice [6, 36]. Moreover, phenotype data available in The International Mouse Phenotyping Consortium (IMPC, https:// www.mousephenotype.org/) show that all female and male homozygous Axin2 knockout $\left(A x i n 2^{-/-}\right)$mice present abnormal head shape malformation. For heterozygous $A x i n 2$ deletion $\left(A x i n 2^{+/-}\right)$mice, a total of 2/9 females develop abnormal head shape at E18.5; 


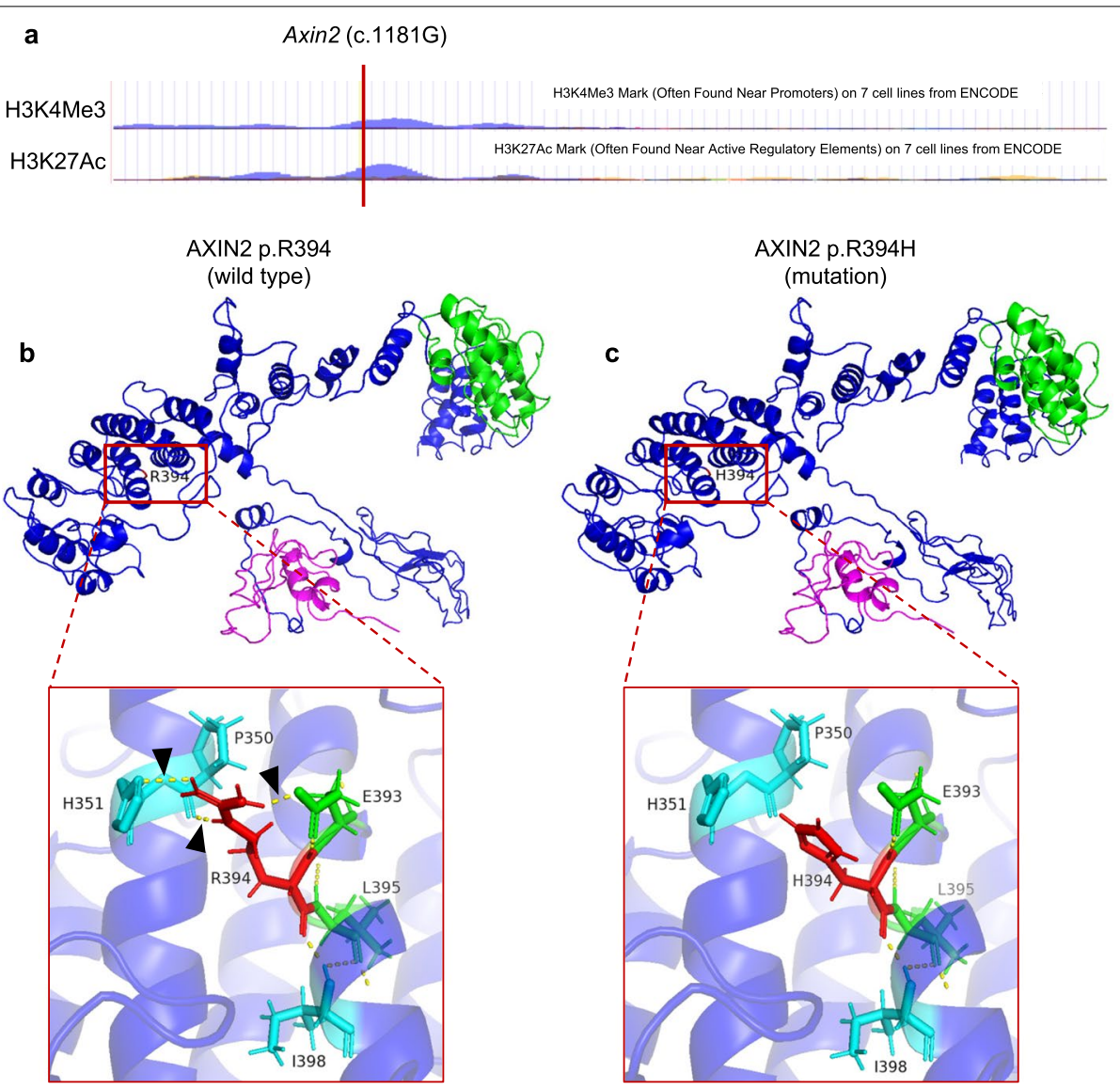

Fig. 5 Functional annotation and structural analysis and of AXIN2 (c.1181G > A: p.R394H, rs200899695). a UCSC database predicted that wild type AXIN2 (c.1181G) loci locates in the region modified by H3K4Me3 or H3K27Ac in seven cell lines by ChIP-Seq assay. b Location of Arg394 residues within the GSK3 $\beta$ binding domain of AXIN2. $\mathbf{c}$ Location of the His 394 residues within the GSK3 $\beta$ binding domain of AXIN2. Arrowheads indicate the hydrogen bond in the domain

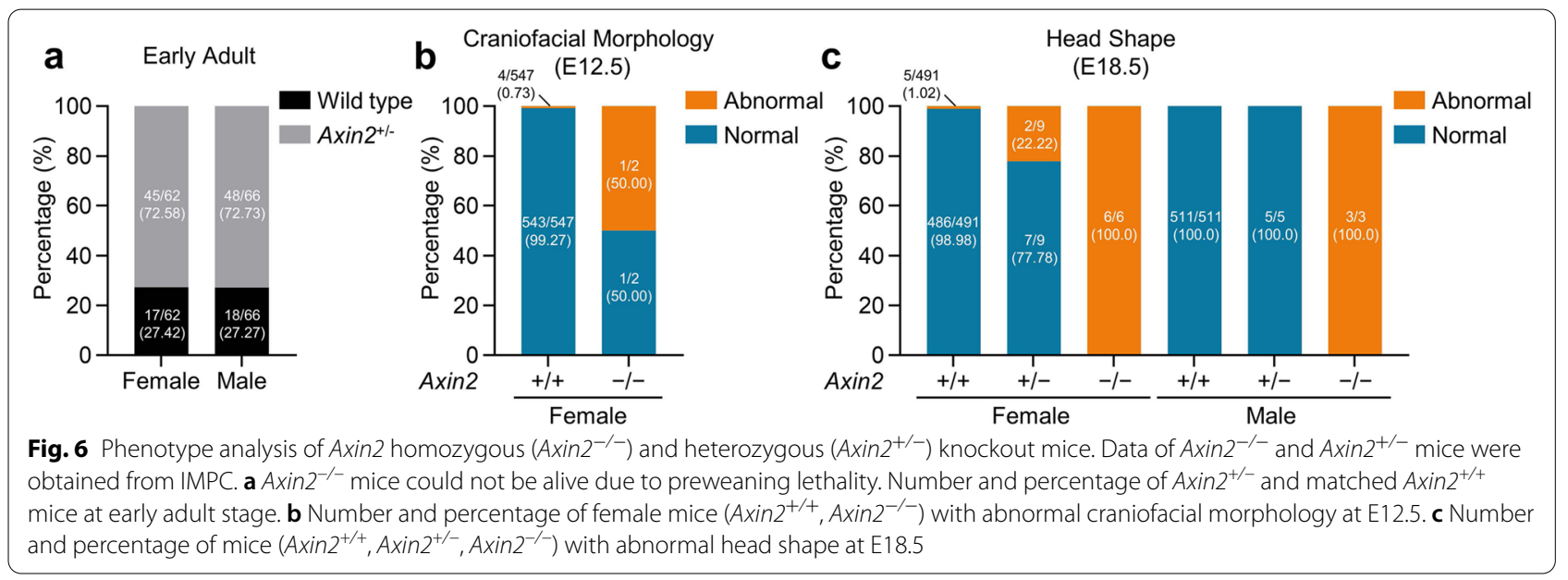


however, the male $A x i n 2^{+/-}$mice are not. These results indicate haploinsufficiency of Axin2 in female mice with incomplete penetrance.

In this study, an Axin 2 heterozygous missense mutation (c.1181G > A: p.R394H, rs200899695) was identified in peripheral blood samples of subjects I-1, II-1 and II-2 (Fig. 1a), suggesting that the proband inherits the mutation from her father. The wild type Axin2 (c.1181G) loci is conserved across species and in the region may be modified by H3K4Me3 or H3K27Ac in seven cell lines by ChIP-Seq assay. H3K4Me3 modification is known to mark genes that are essential for the identity and function. H3K4Me3 breadth contains information that ensures transcriptional precision at the identified genes [38]. H3K27Ac, a robust mark of active enhancers and promoters, has been demonstrated to be strongly correlated with gene expression and transcription-factor binding [39]. However, much more functional annotation and experiments are needed to clarify whether Axin2 (c.1181G > A: p.R394H, rs200899695) could affect the H3K4Me3 or H3K27Ac modification. In addition, Axin2 missense mutation (c.1181G > A: p.R394H, rs200899695) is likely to be deleterious by in silico predication; however, only the proband received additional risk factors (persistent breech presentation and intrauterine growth restriction) developed sagittal craniosynostosis. We assumed that phenotypic segregation in our case was probably due to Axin2 (c.1181G > A) mutation possesses incomplete penetrance, thus making it insufficiency to trigger the disease alone. Our findings corroborate another well-established gene-environment interaction model of NCS, which substantiates the same environmental insults ultimately determining phenotype [15]. However, our gene-environment interaction fashion was observed in the context of Axin2 (c.1181G > A, p.R394H, rs200899695) mutation and female individual, further clinical observations, animal and mechanistic studies are needed to validate the hypothesis.

However, there are some limitations of our study. Experiments on whether Axin2 (c.1181G > A, p.R394H, rs200899695) mutation could affect the H3K4Me3 or H3K27Ac modification of AXIN2 are needed. We only offered a plausible explanation of Axin2 (c.1181G > A, p.R394H, rs200899695) mutation and perinatal risk factors contribute to sagittal craniosynostosis in a Chinese female monochorionic diamniotic twin family. Given the small sample size and inherent ascertainment bias, the identification of further families in the setting of this mutation would help to clarify the clinical implications.

\section{Conclusion}

Based on the results of monochorionic twins, we demonstrated Axin2 (c.1181G > A, p.R394H, rs200899695) mutation led to haploinsufficiency with incomplete penetrance in female, and additional prenatal risk factors (intrauterine growth restriction and breech position) were indispensable to trigger the occurrence of sagittal craniosynostosis in this Chinese female monochorionic diamniotic twin family. These findings provide new evidence for the gene-environment interplay in understanding etiologies of NCS.

\section{Abbreviations \\ Axin2: Axis inhibitor 2; BMP: Bone morphogenic protein; CS: Craniosynostosis; ExAC: Exome Aggregation Consortium; FET: Frozen embryo transfer; FGF: Fibroblast growth factor; FGFR2: Fibroblast growth factor receptor 2; IMPC: The International Mouse Phenotyping Consortium; MAF: Minor allele frequency; MC twins: Monochorionic (MC) twins; MCDA: Monochorionic diamniotic; NCS: Non-syndromic craniosynostosis; TSH: Thyroid-stimulating hormone; TWIST1: Twist, drosophila, homolog of 1; WES: Whole exome sequencing; Wnt: Wingless-type integration sites.}

\section{Supplementary Information}

The online version contains supplementary material available at https://doi. org/10.1186/s41065-021-00182-0.

\section{Additional file 1}

\section{Acknowledgments}

We thank Xi Chen for her kind help in drawing the schematic diagram of fetal position.

\section{Authors' contributions}

AHG, GW, JX designed the study. GW, QY, LZ provided patients' data and performed clinical assessments. JX, CCS, JJL, ZKW, CX, QL, SQX, LYZ, YS conducted the experiments. JX, QY, CCS, JJL, XZ, ZKW analysed the data. JX, QY, CCS, JJL wrote the manuscript. All authors critically revised the manuscript for important intellectual content. AHG, GW supervised the study. The authors read and approved the final manuscript.

\section{Funding}

This work was financially supported by the Opening Foundation of State Key Laboratory of Reproductive Medicine (SKLRM-K202009), National Natural Science Foundation of China (81703261), the Natural Science Foundation of Jiangsu Province (BK20171060), the Natural Science Foundation of the Jiangsu Higher Education Institutions of China (17KJB330002), the Jiangsu Students' Platform for Innovation and Entrepreneurship Training Program (201810312036Y), the Foundation of Priority Academic Program Development (PAPD) of Jiangsu Higher Education Institutions (Public Health and Preventive Medicine), the Top-notch Academic Programs Project of Jiangsu Higher Education Institutions.

Availability of data and materials

All the data generated in the present research is contained in this manuscript.

\section{Declarations}

\section{Ethical approval and consent to participate}

This research was carried out according to the principles of the Declaration of Helsinki and was approved by the Nanjing Medical University Ethics Committee. 


\section{Consent for publication}

Not applicable.

\section{Competing interests}

The authors declare that they have no competing interests.

\begin{abstract}
Author details
${ }^{1}$ State Key Laboratory of Reproductive Medicine, Institute of Toxicology, Nanjing Medical University, Nanjing 211166, China. ${ }^{2}$ Key Laboratory of Modern Toxicology, Ministry of Education, Center for Global Health, School of Public Health, Nanjing Medical University, Nanjing 211166, China. ${ }^{3}$ Department of Maternal, Child and Adolescent Health, School of Public Health, Nanjing Medical University, Nanjing 211166, China. ${ }^{4}$ Department of Neurosurgery, Children's Hospital of Nanjing Medical University, Nanjing 210017, China. ${ }^{5}$ Obstetrics and Gynecology Hospital, NHC Key Laboratory of Reproduction Regulation (Shanghai Institute of Planned Parenthood Research), School of Life Sciences, Shanghai 200011, China. ${ }^{6}$ Department of Neurosurgery, The First Affiliated Hospital of Nanjing Medical University, Nanjing 210029, China.
\end{abstract}

Received: 12 January 2021 Accepted: 27 April 2021

Published online: 16 June 2021

\section{References}

1. Timberlake AT, Persing JA. Genetics of Nonsyndromic Craniosynostosis. Plast Reconstr Surg. 2018;141(6):1508-16.

2. Paumard-Hernandez B, Berges-Soria J, Barroso E, Rivera-Pedroza Cl, PerezCarrizosa V, Benito-Sanz S, Lopez-Messa E, Santos F, Garcia R II, Romance $A$, et al. Expanding the mutation spectrum in 182 Spanish probands with craniosynostosis: identification and characterization of novel TCF12 variants. European journal of human genetics: EJHG. 2015;23(7):907-14.

3. Justice CM, Yagnik G, Kim Y, Peter I, Jabs EW, Erazo M, Ye X, Ainehsazan E, Shi L, Cunningham ML, et al. A genome-wide association study identifies susceptibility loci for nonsyndromic sagittal craniosynostosis near BMP2 and within BBS9. Nat Genet. 2012;44(12):1360-4.

4. Dempsey RF, Monson LA, Maricevich RS, Truong TA, Olarunnipa S, Lam SK, Dauser RC, Hollier LH Jr, Buchanan EP. Nonsyndromic Craniosynostosis. Clin Plast Surg. 2019;46(2):123-39.

5. Durham EL, Howie RN, Cray JJ. Gene/environment interactions in craniosynostosis: A brief review. Orthod Craniofac Res. 2017;20(Suppl 1):8-11.

6. Yilmaz E, Mihci E, Guzel Nur B, Alper OM. A novel AXIN2 gene mutation in sagittal synostosis. Am J Med Genet A. 2018;176(9):1976-80.

7. Sergesketter AR, Elsamadicy AA, Lubkin DT, Krucoff KB, Krucoff MO, Muh CR Characterization of Perinatal Risk Factors and Complications Associated With Nonsyndromic Craniosynostosis. J Craniofac Surg. 2019:30(2):334-8.

8. Miller KA, Twigg SR, McGowan SJ, Phipps JM, Fenwick AL, Johnson D, Wall SA, Noons P, Rees KE, Tidey EA, et al. Diagnostic value of exome and whole genome sequencing in craniosynostosis. J Med Genet. 2017;54(4):260-8.

9. Timberlake AT, Furey CG, Choi J, Nelson-Williams C, Yale Center for Genome A, Loring E, Galm A, Kahle KT, Steinbacher DM, Larysz D et al: De novo mutations in inhibitors of Wnt, BMP, and Ras/ERK signaling pathways in non-syndromic midline craniosynostosis. Proc Natl Acad Sci U S A 2017, 114(35):E7341-E7347.

10. Katsianou MA, Adamopoulos C, Vastardis H, Basdra EK. Signaling mechanisms implicated in cranial sutures pathophysiology: Craniosynostosis. BBA Clin. 2016;6:165-76.

11. Sanchez-Lara PA, Carmichael SL, Graham JM Jr, Lammer EJ, Shaw GM, Ma C, Rasmussen SA. National Birth Defects Prevention S: Fetal constraint as a potential risk factor for craniosynostosis. Am J Med Genet A. 2010;152A(2):394-400.

12. Lee HQ, Hutson JM, Wray AC, Lo PA, Chong DK, Holmes AD, Greensmith AL. Changing epidemiology of nonsyndromic craniosynostosis and revisiting the risk factors. J Craniofac Surg. 2012;23(5):1245-51.

13. James AW, Levi B, XuY, Carre AL, Longaker MT. Retinoic acid enhances osteogenesis in cranial suture-derived mesenchymal cells: potential mechanisms of retinoid-induced craniosynostosis. Plast Reconstr Surg. 2010;125(5):1352-61.

14. Carmichael SL, Rasmussen SA, Lammer EJ, Ma C, Shaw GM. National Birth Defects Prevention S: Craniosynostosis and nutrient intake during pregnancy. Birth Defects Res A Clin Mol Teratol. 2010;88(12):1032-9.
15. Johnson D, Wall SA, Mann S, Wilkie AO. A novel mutation, Ala315Ser, in FGFR2: a gene-environment interaction leading to craniosynostosis? European journal of human genetics : EJHG. 2000;8(8):571-7.

16. Derom C, Thiery E, Rutten BPF, Peeters H, Gielen M, Bijnens E, Vlietinck R, Weyers S: The East Flanders Prospective Twin Survey (EFPTS): 55 Years Later. Twin Res Hum Genet 2019:1-6.

17. Li H, Durbin R. Fast and accurate long-read alignment with Burrows-Wheeler transform. Bioinformatics (Oxford, England). 2010;26(5):589-95.

18. McKenna A, Hanna M, Banks E, Sivachenko A, Cibulskis K, Kernytsky A, Garimella K, Altshuler D, Gabriel S, Daly M, et al. The Genome Analysis Toolkit: a MapReduce framework for analyzing next-generation DNA sequencing data. Genome Res. 2010;20(9):1297-303.

19. Wang K, Li M, Hakonarson H. ANNOVAR: functional annotation of genetic variants from high-throughput sequencing data. Nucleic Acids Res. 2010;38(16):e164.

20. Lek M, Karczewski KJ, Minikel EV, Samocha KE, Banks E, Fennell T, O’DonnellLuria AH, Ware JS, Hill AJ, Cummings BB, et al. Analysis of protein-coding genetic variation in 60,706 humans. Nature. 2016;536(7616):285-91.

21. Kumar P, Henikoff S, Ng PC. Predicting the effects of coding non-synonymous variants on protein function using the SIFT algorithm. Nat Protoc. 2009;4(7):1073-81.

22. Adzhubei IA, Schmidt S, Peshkin L, Ramensky VE, Gerasimova A, Bork P, Kondrashov AS, Sunyaev SR. A method and server for predicting damaging missense mutations. Nat Methods. 2010;7(4):248-9.

23. Schwarz JM, Cooper DN, Schuelke M, Seelow D. MutationTaster2: mutation prediction for the deep-sequencing age. Nat Methods. 2014;11(4):361-2.

24. Ashburner M, Ball CA, Blake JA, Botstein D, Butler H, Cherry JM, Davis AP, Dolinski K, Dwight SS, Eppig JT, et al. Gene ontology: tool for the unification of biology. Gene Ontology Consortium Nature Genet. 2000;25(1):25-9.

25. Kanehisa M, Furumichi M, Tanabe M, Sato Y, Morishima K. KEGG: new perspectives on genomes, pathways, diseases and drugs. Nucleic Acids Res. 2017:45(D1):D353-d361.

26. Crooks GE, Hon G, Chandonia JM, Brenner SE. WebLogo: a sequence logo generator. Genome Res. 2004;14(6):1188-90.

27. Roy A, Kucukural A, Zhang Y. I-TASSER: a unified platform for automated protein structure and function prediction. Nat Protoc. 2010;5(4):725-38.

28. Li X, Li Z, Zhou H, Gaynor SM, Liu Y, Chen H, Sun R, Dey R, Arnett DK, Aslibekyan $\mathrm{S}$, et al. Dynamic incorporation of multiple in silico functional annotations empowers rare variant association analysis of large wholegenome sequencing studies at scale. Nat Genet. 2020;52(9):969-83.

29. Ring N, Meehan TF, Blake A, Brown J, Chen CK, Conte N, Di Fenza A, Fiegel T, Horner N, Jacobsen JO, et al. A mouse informatics platform for phenotypic and translational discovery. Mamm Genome. 2015;26(9-10):413-21.

30. Munoz-Fuentes V, Cacheiro P, Meehan TF, Aguilar-Pimentel JA, Brown SDM, Flenniken AM, Flicek P, Galli A, Mashhadi HH, Hrabe de Angelis M et al: The International Mouse Phenotyping Consortium (IMPC): a functional catalogue of the mammalian genome that informs conservation. Conserv Genet 2018, 19(4):995-1005.

31. Villar J, Giuliani F, Bhutta ZA, Bertino E, Ohuma EO, Ismail LC, Barros FC, Altman DG, Victora C, Noble JA, et al. Postnatal growth standards for preterm infants: the Preterm Postnatal Follow-up Study of the INTERGROWTH-21(st) Project. Lancet Glob Health. 2015;3(11):e681-691.

32. Bendon CL, Sheerin FB, Wall SA, Johnson D. The relationship between scaphocephaly at the skull vault and skull base in sagittal synostosis. J Craniomaxillofac Surg. 2014;42(3):245-9.

33. Ren J, Wen L, Gao X, Jin C, Xue Y, Yao X: DOG 1.0: illustrator of protein domain structures. Cell Res 2009, 19(2):271-273.

34. Lakin GE, Sinkin JC, Chen R, Koltz PF, Girotto JA. Genetic and epigenetic influences of twins on the pathogenesis of craniosynostosis: a meta-analysis. Plast Reconstr Surg. 2012;129(4):945-54.

35. Liu B, Yu HM, Hsu W. Craniosynostosis caused by Axin2 deficiency is mediated through distinct functions of beta-catenin in proliferation and differentiation. Dev Biol. 2007:301(1):298-308.

36. Yu H-MI, Jerchow B, Sheu T-J, Liu B, Costantini F, Puzas JE, Birchmeier W, Hsu W: The role of Axin2 in calvarial morphogenesis and craniosynostosis. Development 2005, 132(8):1995-2005.

37. Maruyama T, Jeong J, Sheu T-J, Hsu W. Stem cells of the suture mesenchyme in craniofacial bone development, repair and regeneration. Nat Commun. 2016:7:10526-10526. 
38. Benayoun BA, Pollina EA, Ucar D, Mahmoudi S, Karra K, Wong ED, Devarajan K, Daugherty AC, Kundaje AB, Mancini E, et al. H3K4me3 breadth is linked to cell identity and transcriptional consistency. Cell. 2014;158(3):673-88.

39. Creyghton MP, Cheng AW, Welstead GG, Kooistra T, Carey BW, Steine EJ, Hanna J, Lodato MA, Frampton GM, Sharp PA, et al. Histone H3K27ac separates active from poised enhancers and predicts developmental state. Proc Natl Acad Sci U S A. 2010;107(50):21931-6.

\section{Publisher's Note}

Springer Nature remains neutral with regard to jurisdictional claims in published maps and institutional affiliations.

- fast, convenient online submission

- thorough peer review by experienced researchers in your field

- rapid publication on acceptance

- support for research data, including large and complex data types

- gold Open Access which fosters wider collaboration and increased citations

- maximum visibility for your research: over $100 \mathrm{M}$ website views per year

At BMC, research is always in progress.

Learn more biomedcentral.com/submissions 